\title{
On the (fuzzy) logical content of CADIAG-2
}

\author{
Agata Ciabattoni \\ Institute for Discrete Mathematics and Geometry \\ Wiedner Hauptstraße 8-10, 1040 Wien, Austria \\ agata@logic.at \\ Thomas Vetterlein ${ }^{1}$ \\ Section on Medical Expert and Knowledge-Based Systems \\ Medical University of Vienna \\ Spitalgasse 23, 1090 Wien, Austria \\ Thomas.Vetterlein@meduniwien.ac.at
}

\begin{abstract}
CADIAG-2 is a successful expert system assisting in the differential diagnosis in internal medicine. With its aid, conjectures about present diseases are derived from possibly vague information about a patient's symptoms. In this paper we provide a mathematical formalisation of the inferential mechanism of CADIAG-2. A Gentzen-style calculus for the resulting logic is introduced and used to compare the system's behaviour with t-norm based fuzzy logics.
\end{abstract}

\section{Introduction}

The last decades have witnessed a considerable development of rule-based systems in medicine with the purpose of assisting physicians in medical decision-making. CADIAG-1 and CADIAG-2 - where "CADIAG" stands for "computer-assisted diagnosis" - are well performing computer-based medical consultation systems whose design and construction was initiated in the early 80's at the Medical University of Vienna by K.-P. Adlassnig; see, e.g., [AdKo, AKSEG, AKSG, LAK]. The aim of these systems is to support diagnostics in the field of internal medicine.

CADIAG-1 deals with Boolean relationships, formulated as if-then rules, between symptoms, signs, laboratory test results and clinical findings on the one hand and diseases on the other hand. A simple and elegant formalisation of CADIAG-1's rules into a decidable fragment of first-order classical logic allowed for consistency checking of the binary rules and the detection of 17 inconsistencies out of the 50000 rules [MoAd].

\footnotetext{
${ }^{1}$ Both authors contributed equally to this paper.
} 
Precise and definite information about real world objects, however, is difficult to obtain and, in the realm of medicine, such information is typically not accessible to physicians when deciding about a patient's diagnosis and treatment. To process vague information, the successor system CADIAG-2 was based on fuzzy set theory, or "fuzzy logic" in Zadeh's terminology [Zad]. A clear understanding of the system's behaviour calls, however, for a mathematical formalisation. This formalisation is also a prerequisite for consistency checking of its knowledge base and for a clear distinction between degrees of presence and degrees of certainty, cf. [DHN].

The aim of this paper is to provide such a formalisation. The result is furthermore used to compare the system's behaviour with the principles of t-norm based logics. We recall that a t-norm is commonly used in fuzzy logic to combine truth degrees in the sense of conjunction. By a t-norm based logic, we mean a propositional logic which uses the real unit interval as the set of truth values and which interprets the conjunction by a (specific kind of) t-norm, see, e.g., [Haj].

Our discussion mainly follows proof theoretical arguments. Indeed, we first introduce CADIAG Logic, or CadL for short, which is modelled upon the mode of operation of CADIAG-2; what CADIAG-2 is able to derive from assumptions can be proved in CadL from the corresponding set of formulas. We relate CadL to a calculus for the fuzzy logic closest to the natural semantical framework for CadL. The latter logic, which we call Gödel-Zadeh logic, or GZL for short, turns out to be a modification of $R G L_{\sim}$, i.e. Gödel logic enriched with standard negation and rational truth constants [EGHN].

The paper is organised as follows. In Section 2 we review the method on which the inference of CADIAG-2 is based. Our description reformulates, in a formally oriented way, the information contained in the thesis [Fis] as well as in a recent implementation of CADIAG-2. Section 3 introduces the logical calculus CadL. We show that each CADIAG-2 run can be simulated by a proof in CadL, while only suitable proofs, which we call updating, correspond to CADIAG-2 runs.

Section 4 meets the challenge to provide a semantical foundation for CadL, and hence for CADIAG-2. In Section 5 we introduce SeqCadL, a Gentzen-style calculus which is sound with respect to the semantics considered. We show that SeqCadL is equivalent to CadL under suitable conditions. Due to the presence of a cut-like rule, SeqCadL is not analytic, that is, its derivations cannot be determined by a simple (and automatable) step-wise decomposition of the statements to be proved. However, SeqCadL is used in Section 6 to compare the CADIAG2 inferential mechanism with GZL, which is the fuzzy logic sound and complete with respect to the natural semantics for CadL. To this aim we introduce an analytic proof system for GZL which uses sequents-of-relations, a generalisation of 
Gentzen's sequents introduced in $[\mathrm{BaFe}]$. As a result of our comparison, we get a rather clear picture about differences and analogies between SeqCadL and GZL, or more generally, between CADIAG-2 and fuzzy logics in the sense of [Haj]. GZL turns out to be strictly stronger than SeqCadL. Indeed the calculus for GZL includes the possibility to consider sets of exhaustive alternatives, whose consequences may be recombined. It is exactly this possibility which is not present in CADIAG-2. This in turn seems not to mirror a weakness, but rather to be a general characteristic of reasoning in medicine, deserving further systematic investigation.

\section{A formal description of CADIAG-2}

The aim of CADIAG-2 may be roughly described as follows. On the basis of a set of symptoms known for some patient, possibly supplemented by certain already established diagnoses, CADIAG-2 is supposed to derive conjectures about the disease(s) of the patient. In a way according to our needs, we will outline how this is done; for further details, see, e.g., [AdKo, Fis].

CADIAG-2 comprises a knowledge base and an inference engine. The former consists of if-then rules describing known causal, statistical, or simply definitional interrelations between symptoms and diagnoses. On the basis of this general information and the information referring to a particular patient, the inference engine can draw conclusions. We note that symptoms and diseases are not analysed with respect to their meaning, but are rather treated as pure propositions; what matters is their mutual relationship.

An example of a proposition referring to a symptom might be "suffering from a strong abdominal pain". It is obvious that the alternatives true and false to evaluate this proposition are not exhaustive. Accordingly, CADIAG- 2 considers all statements about symptoms as vague. Namely, to each symptom, there is associated a degree of presence, expressed by an element of the real unit interval $[0,1]$. The second kind of propositions in CADIAG- 2 refers to diagnoses. Namely, CADIAG2 processes statements expressing that a patient suffers from a specific disease. We do not assume to deal with vagueness in this case; it is assumed that a disease is present or not. However, it is rarely possible to provide a diagnosis with certainty and consequently, in CADIAG-2, to each proposition referring to a diagnosis, there is associated a degree of certainty, which is again an element of $[0,1]$.

The truth values. The meaning of the truth values is understood in CADIAG-2 as follows. Asserting that a symptom $\sigma$ applies to the degree $t \in[0,1]$ means, as usual in fuzzy set theory, that the patient's state is compatible with the property 
expressed by $\sigma$ to the degree $t$. In particular, the case $t=1$ means that $\sigma$ is clearly confirmed; $t=0$ means that $\sigma$ definitely does not hold; and otherwise a smaller or larger choice of $t$ expresses that $\sigma$ applies to a smaller or larger extent. In practice, to determine $t$, we either use the patient's or medical doctor's appraisal how well a subjective observation fits to the symptom; examples of this type of symptoms include "strong abdominal pain" or "suspicion of pancreatic tumour by CT". Or a symptom refers to a measurement, like for example in the case of "having high fever"; then a predefined fuzzy set over the respective scale, like the interval $[36,42]$ of possible body temperatures in ${ }^{\circ} \mathrm{C}$, is used to determine $t$. We finally note that CADIAG-2 also offers the possibility not to assign any truth value to a symptom $\sigma$; this means that we make no assertion about $\sigma$.

In case of a diagnosis $\delta$, the situation is different. To assign a value $t \in(0,1]$ to the diagnosis $\delta$ means that we are certain to the degree $t$ that the disease is present. In particular, in case $t=1$ we assert that the disease is definitely present; and in case $0<t<1$, we are less sure, where a smaller value means a smaller degree of certainty. In case of, say, $t=0.001$, we practically do not assert anything. Furthermore, 0 plays an extra role here. Saying that a diagnosis $\delta$ holds to the degree 0 means that the negation of $\delta$ definitely holds, that is, it means that we are convinced that $\delta$ must be excluded.

We formalise below the concept of strength of a truth value.

Definition 2.1. For $s, t \in[0,1]$, we say that $t$ is stronger than $s$

$$
\text { if either } 0<s \leq t \leq 1 \text { or } 0<s<1 \text { and } t=0 \text {; }
$$

in this case, we write $s \preccurlyeq t$. Furthermore, we call 0 and 1 sharp truth values.

Note that both sharp truth values are maximal w.r.t. $\preccurlyeq$. The reason is that they express definite information which cannot be improved. Note that $\preccurlyeq$ defines not a linear, but just a partial order on the set $[0,1]$ of truth values.

The input and output. Assume that we are given a specific knowledge base. We discuss below the way CADIAG-2 makes use of it. We formalise below the specification given in [Fis].

Let $\sigma_{1}, \ldots, \sigma_{m}$ denote the symptoms and $\delta_{1}, \ldots, \delta_{n}$ the diagnoses appearing in the knowledge base. A symbol denoting either a symptom or a diagnosis will be called a basic entity. We shall identify a symptom $\sigma_{i}$ with the proposition "the symptom $\sigma_{i}$ is present" and, similarly, we will identify a diagnosis $\delta_{j}$ with the proposition "the diagnosis $\delta_{j}$ applies". Furthermore, we can consider compound entities. The connectives which are at our disposal in CADIAG-2 are the conjunction $\wedge$, the 
disjunction $\vee$, and negation $\sim$. By an entity, we shall mean a basic entity, or a compound entity built up by means of $\wedge, \vee, \sim$ from basic ones.

There are two sets of additional connectives called at least $n$ out of $m$ and at most $n$ out of $m$, where $1 \leq n \leq m$. Their use in the medical literature is common when diseases are specified by a set of criteria; cf., e.g., [GJEHH]. These connectives are in the expected way expressible by $\wedge$ and $\vee$ and are therefore not taken into account here. As an example,

$$
\text { at least } 2 \text { of }\left(\varphi_{1}, \varphi_{2}, \varphi_{3}\right)
$$

stands for $\left(\varphi_{1} \wedge \varphi_{2}\right) \vee\left(\varphi_{1} \wedge \varphi_{3}\right) \vee\left(\varphi_{2} \wedge \varphi_{3}\right)$.

Recall that a partial function $f: A \rightarrow B$ is a function from a subset $A_{0}$ of $A$ to $B$. We write $f(a)=b$ to express that $f(a)$ is defined and equals $b$, and we say that $f(a)$ is undefined if $a \notin A_{0}$. $A_{0}$ will be called the domain of $f$.

Henceforth we will denote the operations min, max and $t \mapsto 1-t$, when applied to the set $[0,1]$ of truth values, by $\bar{\wedge}, \bar{\nabla}$ and $\bar{\sim}$, respectively.

Definition 2.2. A partial evaluation, or simply an evaluation, is a partial function $v$ from the set of entities to the real unit interval $[0,1]$ such that, for all entities $\alpha$ and $\beta$, the following holds. (i) If $v(\alpha)=s$ and $v(\beta)=t$, then $v(\alpha \wedge \beta)=s \wedge t$ and $v(\alpha \vee \beta)=s \nabla t$; (ii) if $v(\alpha)=0$ or $v(\beta)=0$, then $v(\alpha \wedge \beta)=0$; (iii) if $v(\alpha)=t>0$ and $v(\beta)$ is undefined, or $v(\alpha)$ is undefined and $v(\beta)=t>0$, then $v(\alpha \vee \beta)=t$; (iv) if $v(\alpha)=t$, then $v(\sim \alpha)=\approx t$; (v) if, for a compound entity $\gamma$, $v(\gamma)$ cannot be determined by means of the rules (i)-(iv), $v(\gamma)$ remains undefined.

Note that an evaluation is uniquely determined by its restriction to the basic entities. Namely, an evaluation assigns truth values to certain, but not necessarily all basic entities; and this assignment is extended to include as many compound entities as possible. As usual, truth functionality applies; a compound proposition is assigned truth values on the basis of the assignment of its components.

Furthermore, Definition 2.2 was chosen in a way to provide as much information as possible about compound propositions on the basis of the available knowledge. The result is not surprising as far as the conjunction and the negation is concerned. The disjunction, however, is treated in a peculiar way. If, e.g., $\alpha$ is assigned 0.3 and the status of $\beta$ is unknown, then $\alpha \vee \beta$ is mapped to 0.3 rather than left undefined. Here, the tacit assumption is made that $\beta$ is not checked for good reason. The special treatment of the disjunction contributes to the effectiveness of CADIAG-2.

The input of a run of CADIAG-2 is an evaluation, which we will refer to as the initial evaluation. Furthermore, we will call the elements of its domain the input 
entities. An initial evaluation describes the state of a patient. Typically, an input entity is a symptom; but it may also refer to a prior established diagnosis.

The output of a run of CADIAG-2 is an evaluation, which we will refer to as the final evaluation. Its domain extends the domain of the initial evaluation.

The inferences. Starting from the initial evaluation $w_{0}$, new evaluations $w_{1}, w_{2}, \ldots$ are obtained by successively applying the rules of the knowledge base; we explain below how a rule is applied. Compared to its predecessor, each evaluation in this sequence encodes an increased amount of information about the patient. The process terminates after finitely many, say $l$, steps; then $w_{l}$ is the final evaluation.

As we will see, the used rule, say $(\mathrm{R})$, influences the truth value of only one specific basic entity $\beta$. So the $k$-th evaluation $w_{k}$ where $k=1, \ldots, l$ will differ from $w_{k-1}$ only in the values assigned to $\beta$ and the compound entities containing $\beta$. On the basis of $w_{k-1},(\mathrm{R})$ provides a truth value $b \in[0,1]$; we call $b$ the proposed truth value for $\beta$. The new evaluation $w_{k}$ is defined as follows: $w_{k}$ coincides with $w_{k-1}$ for all basic entities different from $\beta$. If $\beta$ is not yet in the domain of $w_{k-1}$, we put $w_{k}(\beta)=b$. If otherwise $w_{k-1}(\beta)>0$ and $b>0$, we put $w_{k}(\beta)=w_{k-1}(\beta) \nabla b$. If $w_{k-1}(\beta)=0$ and $b<1$, or if $w_{k-1}(\beta)<1$ and $b=0$, then $w_{k}(\beta)=0$. The case $w_{k-1}(\beta)=0$ and $b=1$ or $w_{k-1}(\beta)=1$ and $b=0$ is considered contradictory; then the process terminates with an error message. Finally, for compound entities, $w_{k}$ is defined according to Definition 2.2.

The rules. Any rule $(\mathrm{R})$ in the knowledge base contains the following information: (i) a possibly compound entity $\alpha$, called the antecedent, (ii) a basic entity $\beta$, called the succedent and (iii) a type. The following types of rules exist:

$\left(\mathrm{c}_{d}\right)$, where $d \in(0,1]$ : Then $(\mathrm{R})$ is said to be of type confirming to the degree $d$. The additional value $d$ is called the confirmability of $\beta$ by $\alpha$. We will say (R) is of type (c) if we do not wish to mention the parameter $d$.

(me): Then (R) is said to be of type mutually exclusive.

(ao): Then (R) is said to be of type always occurring.

The types (c), (me), and (ao) express different ways in which $\alpha$ and $\beta$ are causally or logically related. For the subsequent explanations, let us assume that $\alpha$ is an expression in symptoms and possibly additional diagnoses, and that $\beta$ is a diagnosis.

If the rule $(\mathrm{R})$ is of type $\left(\mathrm{c}_{d}\right)$, then (R) expresses the fact that if the given symptoms and diagnoses fulfil the expression $\alpha$ to a non-zero degree, this is a hint to the diagnosis $\beta$ and this hint is the more serious the larger $d$ is. Namely, if during a run of CADIAG- $2 \alpha$ evaluates to $t>0$, then the truth value for $\beta$ will be $d \wedge t$, unless 
$\beta$ has been already assigned a stronger truth value. In particular, if $\alpha$ is assigned 1 , that is, if $\alpha$ is assumed to be fully true, and if there is nothing else known about $\beta$, then $\beta$ is assigned $d$. (R) is not usable if $\alpha$ is assigned 0 .

So, if $\alpha$ clearly holds, $d$ is the degree of certainty about $\beta$. This implies a way how the parameters $d$ can be determined. Namely, if a sufficiently large patient database is available, $d$ can be taken as the proportion of patients suffering from the disease $\beta$ among the patients for which the statement $\alpha$ clearly holds. We note that the actual values included in CADIAG-2's knowledge base were however determined slightly differently. To make use of the data available from a database in a more comprehensible way, all patients fulfilling $\alpha$ to a non-zero degree were considered; the cardinalities of crisp sets were replaced by cardinalities of fuzzy sets [AKSG].

The example below and the subsequent examples are taken from [AKSG].

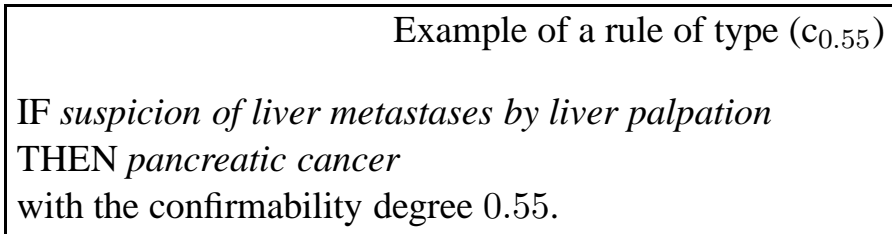

According to our specification, this rule works as follows. Assume that the diagnosis pancreatic cancer was not yet assigned a truth value. If, due to a liver palpation, there is the evident suspicion of liver metastases, we associate to the diagnosis "pancreatic cancer" a degree of certainty of 0.55 . So we assume that the patient has pancreatic cancer, where however a value of 0.55 expresses considerable uncertainty about this conclusion.

If the mentioned suspicion is not so clear, but still quantified by a value of at least 0.55 , the result will be the same.

If a suspicion is present, but to a degree of less than 0.55 , say 0.2 , then the degree of certainty about the diagnosis "pancreatic cancer" will be degraded to 0.2 .

If there is evidently no suspicion of metastases, the rule will not be used.

If $(\mathrm{R})$ if of type (me), then $(\mathrm{R})$ expresses the fact that the two statements exclude each other. In particular, (R) says that if $\alpha$ is fully true, $\beta$ definitely does not apply, i.e. the truth value for $\beta$ is 0 .

Example of a rule of type (me)

IF positive rheumatoid factor

THEN NOT seronegative rheumatoid arthritis

If the positive rheumatoid factor is evaluated 1, then seronegative rheumatoid arthritis is assigned 0. 
If the positive rheumatoid factor is not evaluated 1 , the rule is not used.

Finally, let (R) be of type (ao). Then (R) expresses the fact that $\beta$ implies $\alpha$. It follows that if $\alpha$ is excluded, $\beta$ definitely does not apply. So, if $\alpha$ is evaluated $0, \beta$ is assigned 0 as well.

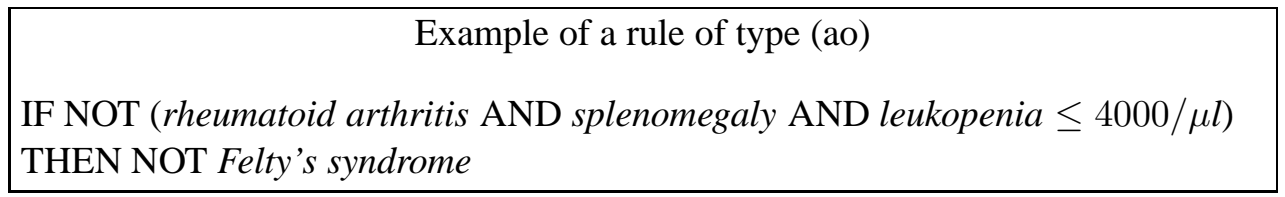

Assume that the expression in brackets, following "IF NOT", is evaluated 0; this means that either rheumatoid arthritis, splenomegaly or leukopenia $\leq 4000 / \mu l$ is fully excluded. Then Felty's syndrome is excluded as well, that is, assigned the value 0 .

If the expression in brackets is evaluated to a non-zero value, the rule is not used.

All three types of rules are also used to express relationships between other kinds of entities, namely: between two symptoms; or between two diagnoses. The meaning and the use of the rules is analogous. In case of a rule of type (c), however, the confirmability is then always 1 .

Termination. The rules are applied systematically one by one, but, as we assume here, in an arbitrary order. The process is completed if, by use of any of the rules, the evaluation remains unchanged. The termination of the program is guaranteed. To see this, note the following. The number of truth values which appear somewhere in the course of the run of CADIAG-2 is limited; we deal with the truth values of finitely many symptoms and finitely many rules, and only these truth values together with their negation can appear. Furthermore, when after each application of a rule the truth value of some entity is renewed the new value is, w.r.t. $\preccurlyeq$, at least as strong as the prior one; so our assertion follows.

\section{CadL - the logical counterpart of CADIAG-2}

In this section we introduce CadL ("CADIAG logic"), a new formal system which, in a specific restricted sense, will turn out to be sound and complete with respect to inferences in CADIAG-2.

Recall that CADIAG-2 deals with entities - i.e. symptoms and diagnoses - with associated truth values. Accordingly, CadL uses what has been called evaluated formulas [Pav, NPM] or graded formulas, [Haj, Ger]: pairs consisting of a proposition and a rational truth value. 
Definition 3.1. The set $\mathcal{F}_{A}$ of atomic propositions of CadL consists of countably many symbols $\varphi_{1}, \varphi_{2}, \ldots$. The set $\mathcal{F}_{L}$ of lattice propositions of CadL consists of the expressions built up from the atomic propositions by means of the binary connectives $\wedge$ and $\vee$ and the unary connective $\sim$. Moreover, let the set $\mathcal{F}_{I}$ of implications of CadL consist of the expressions $\alpha \rightarrow \beta$, where $\alpha, \beta \in \mathcal{F}_{L}$. Finally, $\mathcal{F}=\mathcal{F}_{L} \cup \mathcal{F}_{I}$ is the set of propositions of CadL.

A graded proposition is a pair $(\varphi, t)$ consisting of a proposition $\varphi \in \mathcal{F}$ and a rational value $t \in[0,1]$.

The atomic propositions are meant to correspond to basic entities in CADIAG-2. It is not necessary to make a formal distinction between symptoms and diagnoses; they are, after all, treated in the same way during the inferences in CADIAG-2. Similarly, there is no need to formally distinguish between degrees of presence and degrees of certainty. Moreover, the compound entities in CADIAG-2 translate to lattice propositions of CadL. Finally an entity together with its image under an evaluation corresponds to a graded proposition in CadL.

A rule of CadL is a pair consisting of one or two graded propositions called the assumption(s) and one graded proposition called the conclusion, notated one upon the other and separated by a horizontal line. The rules for CadL are divided into evaluation and manipulation rules: the former serve to determine the truth value associated to a compound proposition, truth values of the atoms being given. The manipulation rules mirror the three kinds of entries in the CADIAG-2 knowledge base: (c), (ao), and (me).

Definition 3.2. The rules of CadL are divided into two groups. The evaluation rules are

$$
\begin{array}{ccc}
\left(\wedge_{1}\right) \frac{(\alpha, s)(\beta, t)}{(\alpha \wedge \beta, s \bar{\wedge} t)} & \left(\wedge_{2}\right) \frac{(\alpha, 0)}{(\alpha \wedge \beta, 0)} & \left(\wedge_{3}\right) \frac{(\beta, 0)}{(\alpha \wedge \beta, 0)} \\
\left(\vee_{1}\right) \frac{(\alpha, s)(\beta, t)}{(\alpha \vee \beta, s \bar{\vee} t)} & \left(\vee_{2}\right) \frac{(\alpha, q)}{(\alpha \vee \beta, q)} & \left(\vee_{3}\right) \frac{(\beta, q)}{(\alpha \vee \beta, q)} \\
(\sim) \frac{(\alpha, t)}{(\sim \alpha, \approx t)} &
\end{array}
$$

for any $\alpha, \beta \in \mathcal{F}_{L}$ and $s, t \in[0,1], q \in(0,1]$.

The manipulation rules are

$$
\text { (c) } \frac{(\alpha \rightarrow \varphi, d) \quad(\alpha, t)}{(\varphi, d \wedge t)} \text { where } d, t>0
$$




$$
\text { (me) } \frac{(\alpha \rightarrow \sim \varphi, 1) \quad(\alpha, 1)}{(\varphi, 0)} \quad \text { (ao) } \frac{(\sim \alpha \rightarrow \sim \varphi, 1) \quad(\alpha, 0)}{(\varphi, 0)}
$$

for any $\alpha, \varphi \in \mathcal{F}_{L}$ such that $\varphi$ is atomic.

A theory of CadL is a finite set $\mathcal{T}$ of graded propositions. A proof from $\mathcal{T}$ is a finite sequence of graded propositions each of which is either in $\mathcal{T}$ or the conclusion of a rule whose assumptions are among the preceding elements of the proof. The $i$-th element of a proof will be called the $i$-th proof entry. The number of entries in a proof is the proof length.

For a lattice proposition $\alpha$ and any $t \in[0,1]$, we call $(\alpha, t)$ provable from $\mathcal{T}$ if there is a proof such that $(\alpha, t)$ is its last entry; in this case, we write $\mathcal{T} \vdash(\alpha, t)$.

Remark 3.3. Note that the rule (c) is very similar to the rule known in fuzzy logic as the fuzzy modus ponens, see, e.g., [NPM, Ger].

We next explain how the case is treated when a proposition appears in a proof with two different truth values.

Definition 3.4. Let $\mathcal{T}$ be a theory of CadL, and let $\alpha \in \mathcal{F}_{L}$. If $\mathcal{T} \vdash(\alpha, 1)$, we say that $\mathcal{T}$ confirms $\alpha$; if $\mathcal{T} \vdash(\alpha, 0)$, we say that $\mathcal{T}$ excludes $\alpha$.

$\mathcal{T}$ is called inconsistent if, for some $\varphi \in \mathcal{F}_{L}, \mathcal{T}$ both confirms and excludes $\varphi$. Otherwise, we say that $\mathcal{T}$ is consistent.

By restricting to consistent theories we will exclude in the sequel the case that, for some lattice proposition $\alpha$, both $(\alpha, 0)$ and $(\alpha, 1)$ is provable. However, it may well happen that $\mathcal{T}$ proves both, say, $(\varphi, 0.6)$ and $(\varphi, 0.7)$; this is not considered a contradiction. In view of the partial order $\preccurlyeq$ of truth values, we rather consider the latter statement stronger than the former. Similarly, $\mathcal{T}$ may prove both $(\varphi, 0)$ and $(\varphi, 0.9)$; this is not considered contradictory either. In this case, $(\varphi, 0)$ is considered stronger because it encodes the definite statement that $\varphi$ is to be excluded. Note, however, that by now, we have not formalised the strength of truth values within our calculus CadL.

We are going to compare the CADIAG-2 inference mechanism and proofs in CadL. Our aim is to establish a one-to-one correspondence; as we shall see, this can be done by adding natural conditions to the way proofs in CadL are made. These conditions will depend on the concept of strength of truth values.

Assume that we are given a fixed CADIAG-2 knowledge base consisting of entries of type (c), (me) or (ao), according to our explanations in Section 2. Let us identify each basic entity appearing in the knowledge base with a unique atomic proposition of CadL, and each compound entity with the respective lattice proposition of CadL. 
Let $v$ be the initial evaluation of a run of CADIAG-2, which is a partial mapping from $\mathcal{F}_{A}$ to $[0,1]$. With $v$, we associate the theory $\mathcal{T}_{v}$ of CadL consisting of the following graded propositions:

(i) $(\varphi, v(\varphi))$ whenever $\varphi \in \mathcal{F}_{A}$ is in the domain of $v$;

(ii) $(\alpha \rightarrow \varphi, d)$, where $d \in(0,1]$, for each rule in the knowledge base of type $\left(\mathrm{c}_{d}\right)$ with antecedent $\alpha$ and succedent $\varphi$;

(iii) ( $\alpha \rightarrow \sim \varphi, 1$ ) for each rule in the knowledge base of type (me) with antecedent $\alpha$ and succedent $\varphi$.

(iv) ( $\sim \alpha \rightarrow \sim \varphi, 1)$ for each rule in the knowledge base of type (ao) with antecedent $\alpha$ and succedent $\varphi$;

Recall that in (ii)-(iv), the proposition $\varphi$ is atomic; so an implication in $\mathcal{T}_{v}$ can be uniquely identified as originating either from a rule of type (c) or from a rule of one of the types (me), (ao).

Completeness. We show how to translate a run of CADIAG-2, starting from the initial evaluation $v$, into a proof of CadL from the theory $\mathcal{T}_{v}$.

Proposition 3.5. Let $\beta$ be any entity in the domain of the final evaluation $w_{l}$ of a run of CADIAG-2. Then, identifying $\beta$ with the corresponding lattice proposition in $\mathrm{CadL},\left(\beta, w_{l}(\beta)\right)$ is provable in $\mathrm{CadL}$ from $\mathcal{T}_{v}$.

Proof. Let a run of CADIAG- 2 be given. Let $w_{0}, \ldots, w_{l}$ be the associated sequence of evaluations, where $w_{0}=v$. Let $1 \leq k \leq l$ and assume that we have already built a proof in CadL from $\mathcal{T}_{v}$ such that, for each basic entity $\varphi$ in the domain of $w_{k-1},\left(\varphi, w_{k-1}(\varphi)\right)$ is contained in $\mathcal{T}_{v}$ or is an entry in the proof.

In the $k$-th step of the run of CADIAG-2, a rule (R) of type (c), (me), or (ao) from the knowledge base is applied; let $\alpha$ be its antecedent and $\varphi$ its succedent. Then $\alpha$ is in the domain of $w_{k-1}$. Let $b$ be the proposed truth value for $\varphi$ produced by the rule, according to the explanations given in Section 2. We will extend the proof in CadL such that $(\varphi, b)$ is its last entry.

In a first step we extend the proof to derive $\left(\alpha, w_{k-1}(\alpha)\right)$; by induction hypothesis this can be done by means of the evaluation rules. Next, we apply one of the three manipulation rules (c), (me), or (ao), corresponding to the type of (R), to derive $(\varphi, b)$.

It follows that there is a proof in CadL from $\mathcal{T}_{v}$ containing $\left(\psi, w_{k}(\psi)\right)$ for each atomic proposition $\psi$ in the domain of $w_{k}$. 
By induction, there is a proof containing $\left(\psi, w_{l}(\psi)\right)$ for each atom $\psi$ occurring in $\beta$. If $\beta$ is not atomic we apply the evaluation rules to derive $\left(\beta, w_{l}(\beta)\right)$.

Soundness. The converse direction is more delicate as not all the proofs in CadL correspond to a run of CADIAG-2. The reason is that when a new value is computed during a run of CADIAG-2, the previously obtained value for the same entity may become obsolete, in which case the old value is discarded. Namely, to emulate the actual mode of operation of CADIAG-2 in CadL, the following properties of CADIAG-2 must be kept:

(C1) If, at the beginning of the $k$-th step, we have for some entity $\beta$ that $w_{k-1}(\beta)=$ $t$, but the rule applied in this step gives $t^{\prime}$ as a truth value for $\beta$, then in all subsequent steps, only one of these values can be used, namely 0 if $t^{\prime}=0$ or $t=0$, and $t \bar{\nabla} t^{\prime}$ if $t, t^{\prime}>0$.

(C2) When the $k$-th step has settled $w_{k}(\varphi)=t$ for a certain basic entity $\varphi$, then the truth value assigned to any entity containing $\varphi$ needs to be updated before being used again.

Let us contrast these conditions with CadL on the basis of an example.

Example 3.6. Consider the following CADIAG-2 rules, say $\left(\mathrm{R}_{1}\right),\left(\mathrm{R}_{2}\right)$, and $\left(\mathrm{R}_{3}\right)$, expressed as graded propositions of $\mathrm{CadL}$ as follows:

$$
\begin{array}{ll}
\left(\mathrm{R}_{1}\right) & \left(\sigma_{1} \rightarrow \delta_{1}, 0.25\right) \\
\left(\mathrm{R}_{2}\right) & \left(\sigma_{2} \rightarrow \sim \delta_{1}, 1\right) . \\
\left(\mathrm{R}_{3}\right) & \left(\sigma_{3} \wedge\left(\delta_{1} \vee \ldots \vee \delta_{7}\right) \rightarrow \delta_{8}, 0.8\right)
\end{array}
$$

Here, $\sigma_{1}$ means aldolase (serum) highly increased and $\delta_{1}$ denotes dermatomyositis. Furthermore, $\sigma_{2}$ means chorea minor; $\sigma_{3}$ means xerostomia; and $\delta_{8}$ denotes arthritis with Sjögren's syndrome. The symbols $\delta_{2}, \ldots, \delta_{7}$ denote some further diseases.

Assume to have the assumptions $\left(\sigma_{1}, 0.7\right),\left(\sigma_{2}, 1\right)$, and $\left(\sigma_{3}, 0.8\right)$. Then a proof in CadL can result in the following situation. Using $\left(\mathrm{R}_{1}\right)$, we derive $\left(\delta_{1}, 0.25\right)$ and using $\left(\mathrm{R}_{2}\right)$ we get $\left(\delta_{1}, 0\right)$. So we have that dermatomyositis is both confirmed with certainty 0.25 and excluded. Now, we may apply $\left(\mathrm{R}_{3}\right)$ using the former result, so 
that we derive $\left(\delta_{8}, 0.25\right)$, that is, arthritis with Sjögren's syndrome with certainty 0.25 .

However, $\left(\delta_{1}, 0\right)$ is a definite statement and $\left(\delta_{1}, 0.25\right)$ involves uncertainty; so the former statement dominates over the latter one. However, the statement about arthritis relies on the statement $\left(\delta_{1}, 0.25\right)$.

The conditions $(\mathrm{C} 1)$ and $(\mathrm{C} 2)$ prevent this unintended situation. Namely, $(\mathrm{C} 1)$ implies that the truth value 0.25 of $\delta_{1}$ becomes obsolete once $\delta_{1}$ is assigned 0 , and (C2) makes sure that the expression $\sigma_{3} \wedge\left(\delta_{1} \vee \ldots \vee \delta_{7}\right)$ is evaluated again if one of the truth values of the contained entities has changed. Consequently, $\left(\mathrm{R}_{3}\right)$ can no longer be used, hence a statement about $\delta_{8}$ is impossible.

We strengthen the notion of a proof in CadL so as to get the exact analogue of the mode of operation of CADIAG-2.

Definition 3.7. Call a proof of CadL from a theory $\mathcal{T}$ updating if the following conditions are fulfilled.

(Up1) Let the $i$-th proof entry be derived by a rule among whose assumptions is $(\alpha, t)$, where $\alpha \in \mathcal{F}_{L}$ and $t \in(0,1)$. If any of the proof entries $1, \ldots, i-1$ or any element of $\mathcal{T}$ is of the form $\left(\alpha, t^{\prime}\right)$, then $t^{\prime} \preccurlyeq t$.

(Up2) Let the $i$-th proof entry be derived by a rule among whose assumptions is $(\alpha, t)$, where $\alpha \in \mathcal{F}_{L}$ is non-atomic. Then, for some $j<i$, the $j$-th proof entry is $(\alpha, t)$ and none of the proof entries $j+1, \ldots, i-1$ is of the form $(\varphi, s)$, where $\varphi$ is an atom occurring in $\alpha$.

For a lattice proposition $\alpha$ and any $t \in[0,1]$, we call $(\alpha, t)$ strictly provable from $\mathcal{T}$ if there is a updating proof such that $(\alpha, t)$ is its last element.

In the evident way, conditions (Up1) and (Up2) reflect the behaviour of CADIAG2 ; they correspond to the conditions $(\mathrm{C} 1)$ and $(\mathrm{C} 2)$ above.

Proposition 3.8. Let $\beta$ be an entity, and let $(\beta, t)$, where $\beta$ is viewed as a lattice proposition in $\mathrm{CadL}$, be strictly provable from $\mathcal{T}_{v}$ in $\mathrm{CadL}$. Then there is a run of CADIAG-2 with $l$ steps such that $w_{l^{\prime}}(\beta)=t$ for some $l^{\prime} \leq l$, where $w_{l^{\prime}}$ is the evaluation after the $l^{\prime}$-th step.

Proof. We translate below any updating proof $P$ in CadL from the theory $\mathcal{T}_{v}$ into inferences of CADIAG-2. Let $m$ be the length of such proof; for each $i=0, \ldots, m$ and each basic entity $\varphi$, we put

$T_{i}^{\varphi}=\left\{t \in[0,1]:(\varphi, t)\right.$ is the $j$-th proof entry for some $j \leq i$, or $(\varphi, t)$ is in $\left.\mathcal{T}_{v}\right\} ;$ 
and, provided that $\mathcal{T}_{v}$ is consistent, we define an evaluation $v_{i}$ which maps a basic entity $\varphi$ to the strongest element of $T_{i}^{\varphi}$ if $T_{i}^{\varphi}$ is non-empty, and which leaves $v_{i}(\varphi)$ undefined otherwise (cf. Definition 2.1). Note that $v_{0}$ coincides with CADIAG-2's initial evaluation $v$. Let $n_{0}=0$, and let $n_{1}, \ldots, n_{l^{\prime}} \in\{1, \ldots, m\}$ be the entries in $P$ which are the result of a usage of a manipulation rule.

We translate $P$ to a CADIAG-2 run in a way that $v_{n_{0}}, v_{n_{1}}, \ldots, v_{n_{l^{\prime}}}$ are the associated evaluations. Namely, assume that, for $1 \leq k \leq l^{\prime}$, we have already translated the proof entries $1, \ldots, n_{k-1}$ of $P$ to $k-1$ CADIAG-2 steps and that the evaluation $w_{k-1}$ of CADIAG-2 after step $k-1$ coincides with $v_{n_{k-1}}$. The $n_{k}$-th proof step is by assumption based on a manipulation rule; let $(\alpha \rightarrow \varphi, d)$ and $(\alpha, t)$ be its assumption and let $(\varphi, s)$ be its conclusion. Then the graded implication is in $\mathcal{T}_{v}$ and thus corresponds to a rule of the CADIAG-2 knowledge base. Furthermore, if $\alpha$ is not atomic, the value $t$ is determined by application of evaluation rules applied to the atoms of $\alpha$. Conditions (Up2) and (Up1) make sure that the truth values used coincide with those given by $w_{k-1}$. So CADIAG- 2 can do an inference reflecting the proof steps $n_{k-1}+1, \ldots, n_{k}$ of $P$, and it follows that $w_{k}=v_{n_{k}}$.

By summarising translating a run of CADIAG- 2 to CadL is straightforward, whereas the translation of a proof in CadL to a CADIAG-2 run is possible only under the condition that at each step the values used in the proof are the "right" - the "updated" - ones; in other words it works only for updating proofs.

\section{Towards a semantical basis for CadL}

We have demonstrated the mutual correspondence of runs of CADIAG-2 and updating proofs of the logic CadL. In this section we pose the question if CadL can be endowed with a semantical basis. We will see that this is the case for the manipulation rules while suitable restrictions on the considered theories are needed to deal with the evaluation rules. Under these restrictions, the conditions (Up1) and (Up2) for updating proofs can be weakened.

The semantics for CadL which we are going to consider, is the one which suggests itself. Namely, we make the natural choice about how to model the propositions corresponding to medical entities: lattice propositions are modelled by truth values, that is, by real numbers between 0 and 1 . The connectives are interpreted in accordance with Definition 2.2: $\wedge$ by the minimum, $\vee$ by the maximum, and $\sim$ by the standard negation. Finally, a graded proposition

$$
(\alpha, t), \quad \text { where } \alpha \in \mathcal{F}_{L} \text { and } t \in(0,1] \text {, }
$$


is, apparently, most naturally understood as "the truth value of $\alpha$ is at least $t$ ". This interpretation is not only in accordance to what is common in fuzzy logics; see, e.g., [Pav, NPM, Haj]. It is actually implied by the concepts underlying CADIAG-2; recall that a truth value $t \in(0,1)$ is any time improvable to a larger one $t^{\prime} \in(0,1]$, in which case the former value is no longer used. Furthermore, 0 plays a special role; a graded proposition $(\alpha, 0)$ where $\alpha \in \mathcal{F}_{L}$, reflects that $\alpha$ is excluded, and it is understood as "the truth value of $\alpha$ is 0 ".

We will first discuss the case that propositions are endowed with sharp truth values and afterwards the case that non-sharp truth values occur.

Definition 4.1. A graded proposition $(\alpha, t)$ is called sharp if $t$ is a sharp truth value. $(\alpha, t)$ is called fuzzy otherwise.

\subsection{Sharp propositions}

We start our discussion by addressing the first, and actually already heavy, obstacle to our aim of a semantical basis for CADIAG-2: the special role played by the truth value 0 . It is a special feature of CADIAG-2 that sharp truth values dominate over fuzzy ones. The particular role assigned to 0 cannot be easily coped with by semantic means; instead, we propose to evade the problem by proof-theoretical means.

We will consider proofs in CadL, to be called sharp-first proofs, which first derive the statements of the form $(\alpha, 0)$ or $(\alpha, 1)$ and only then the rest. A situation like the one described in Example 3.6 will be excluded. Furthermore, as we will see, sharp propositions possess proofs of this form if they possess a proof at all.

Definition 4.2. A proof $P$ of CadL from a theory $\mathcal{T}$ is called sharp-first if $P$ is the concatenation of two sequences $P_{s}$ and $P_{f}$ such that (i) $P_{s}$ contains only sharp propositions, (ii) for any entry in $P_{f}$ of the form $(\alpha, t)$, where $\alpha \in \mathcal{F}$, we have that $\alpha$ does not appear in $P_{s}$ and $t$ is not sharp.

In other words, we require proofs in CadL to be divided into two parts: a first one concerning sharp propositions only, and interpretable in classical propositional logic; and a subsequent one where only fuzzy propositions are inferred.

Example 4.3. To illustrate the effect of this condition, recall Example 3.6. We considered the situation that both $\left(\delta_{1}, 0\right)$ and $\left(\delta_{1}, 0.25\right)$ occurred in a proof, where $\delta_{1}$ denotes a specific diagnosis. In a sharp-first proof this situation is excluded: the sharp propostion $\left(\delta_{1}, 0\right)$ must be derived first; but afterwards an entry $\left(\delta_{1}, 0.25\right)$ is impossible as the application of any rule with $\delta_{1}$ as its conclusion is not allowed. 
We furthermore note that if an inconsistency is detected in a sharp-first proof, meaning that an entity is assigned both 0 and 1 , this happens necessarily in the first part, i.e. in $P_{s}$ w.r.t. the notation of Definition 4.2.

The notion of a sharp-first proof makes sense only if for a sharp proposition provable from $\mathcal{T}_{v}$ also a sharp-first proof exists. This is indeed the case; even better, in sharp-first proofs of sharp propositions, the conditions (Up1) and (Up2) always hold.

Lemma 4.4. Let $\mathcal{T}$ be a consistent theory of $\mathrm{CadL}$ and $(\alpha, b)$ a sharp proposition. If $(\alpha, b)$ is provable from $\mathcal{T}$, then there is a proof of $(\alpha, b)$ from $\mathcal{T}$ in which all graded propositions are sharp. In particular, this proof is sharp-first.

Furthermore, any sharp-first proof of a sharp proposition from $\mathcal{T}$ is an updating proof.

Proof. If a sharp proposition is the conclusion of an evaluation rule, either both assumptions are sharp as well, or one is sharp and the other one can be discarded from the proof. If a sharp proposition is the conclusion of a manipulation rule, then evidently the two assumptions are sharp as well. We conclude that if $(\alpha, b)$ is provable from $\mathcal{T},(\alpha, b)$ can be proved using sharp propositions only. Naturally, this proof is sharp-first.

Furthermore, any sharp-first proof ending with a sharp proposition contains only sharp propositions. In view of the consistency assumption about $\mathcal{T}$, such a proof is always updating.

Corollary 4.5. Let $\mathcal{T}$ be a consistent theory of CadL and $(\alpha, b)$ a sharp proposition. Then $(\alpha, b)$ is provable from $\mathcal{T}$ if and only if $(\alpha, b)$ is strictly provable from $\mathcal{T}$.

In contrast to sharp propositions, a fuzzy proposition may be strictly provable, but might not possess a sharp-first proof.

Example 4.6. Let $\mathcal{T}=\left\{(\varphi, 0.6),(\varphi, 0),\left(\varphi \rightarrow \psi_{1}, 0.7\right),\left(\sim \varphi \rightarrow \sim \psi_{2}, 1\right),\left(\psi_{1} \wedge\right.\right.$ $\left.\left.\sim \psi_{2} \rightarrow \chi, 0.8\right)\right\}$ be a theory of CadL. Then $(\chi, 0.6)$ is provable from $\mathcal{T}$, but a proof necessarily contains both $(\varphi, 0.6)$ and $(\varphi, 0)$ and thus cannot be sharp-first.

In view of Examples 3.6 and 4.6, we conclude that requiring proofs of CadL to be sharp-first means an improvement in transparency. Accordingly, we propose the following modification of CADIAG-2:

(1) Restrict the initial evaluation to those basic entities to which 0 or 1 is assigned and calculate the consequences. If an inconsistency occurs, quit. 
(2) Extend the resulting evaluation to cover also those basic entities of the initial evaluation to which non-sharp truth values are assigned; ignore all rules from the knowledge base whose succedent is already evaluated by 0 or 1 and calculate the consequences until there is no more change.

Call a run of CADIAG-2 subject to these requirements a sharp-first run. Then Propositions 3.5 and 3.8 may be modified in the obvious way, to establish a one-toone correspondence between sharp-first runs of CADIAG- 2 on the one hand and sharp-first updating proofs of CadL on the other hand.

\subsection{Fuzzy propositions}

The semantic interpretation proposed at the beginning of the section is adequate for all rules when restricted to sharp values but it is not for the rule $(\sim)$ for negation when fuzzy values are involved. Indeed, if $t \in(0,1]$ is a lower bound for the truth value of $\alpha$, then, taking into account the interpretation of $\sim$ by $[0,1] \rightarrow$ $[0,1], \quad t \mapsto 1-t$, we conclude that $1-t$ is an upper bound of the truth value of $\sim \alpha$ rather than a lower bound. This problem does not arise anymore if we make the assumptions below. These assumptions are desirable from a logical point of view, but are certainly restrictive from the point of view of the intended application.

Definition 4.7. A proof in CadL from a theory $\mathcal{T}$ is called regular if the following condition holds. Let $(\alpha, t)$ be the assumption of a rule $(\sim)$, and let $\varphi$ be an atom appearing in $\alpha$. Then either $(\varphi, s)$ is contained in $\mathcal{T}$ for some $s \in[0,1]$ or $(\varphi, s)$ is contained in the proof for some $s \in\{0,1\}$.

Furthermore, a theory $\mathcal{T}$ of $\mathrm{CadL}$ is called assumption-preserving if whenever $(\alpha, t) \in \mathcal{T}$ for some $\alpha \in \mathcal{F}_{L}$ and we can prove $\left(\alpha, t^{\prime}\right)$ from $\mathcal{T}$, then $t^{\prime}=t$.

Note that whenever the rule $(\sim)$ is used in a regular proof, we know that, w.r.t. our proposed semantical interpretation, the conclusion gives the exact truth value of the concerned negated proposition, not only a bound from above or below. Regularity is the key condition to endow CadL with a semantical basis.

Moreover, the condition for a theory to be assumption-preserving reflects a natural requirement, namely, that assumptions made should be taken as granted and not be "corrected".

By using the conditions above the notion of updating proof is not needed anymore.

Lemma 4.8. Let $\mathcal{T}$ be an assumption-preserving consistent theory of CadL and $\alpha$ be atomic. Assume that there is a regular proof of $(\alpha, t)$, where $0<t<1$, from $\mathcal{T}$ such that the following condition is fulfilled: 
(Up1') If the $i$-th proof entry is derived by a rule among whose assumptions is $(\varphi, t), \varphi \in \mathcal{F}_{L}, t>0$, then neither of the proof entries $1, \ldots, i-1$ nor of the elements in $\mathcal{T}$ is of the form $(\varphi, 0)$.

Then there is a $t^{\prime} \geq t$ such that there is an updating regular proof of $\left(\varphi, t^{\prime}\right)$ from $\mathcal{T}$.

Proof. Let $P$ be a proof of $(\alpha, t)$, where $t \in(0,1)$, such that (Up1') is fulfilled. We modify the proof as follows.

Assume that we have modified the proof up to the $i-1$-th step, $i \geq 1$, such that up to this point, conditions of updating are fulfilled, and certain entries $(\beta, r)$, where $\beta \in \mathcal{F}_{L}$ and $r \in(0,1)$, in the original proof have been changed to $\left(\beta, r^{\prime}\right), r^{\prime}>r$. Consider now the subsequent use of a manipulation rule.

If the conclusion is sharp, we know by the proof of Lemma 4.4 how the conditions of updating can be achieved.

Otherwise, the manipulation rule is of type (c). Let $(\gamma, s)$ be its right premise. $s$ is the result of an evaluation of $\gamma$ from the truth values of its atoms. The conditions of updating are fulfilled if for each atom, the best truth value appearing in the proof so far, is used. If an atom $\varphi$ appearing in $\gamma$ is in the scope of a negation, then its truth value is sharp and thus maximal w.r.t. $\preccurlyeq$, or it originates from $\mathcal{T}$ and is thus unique in the proof.

If, however an atom $\varphi$ appearing in $\gamma$ is not in the scope of a negation, it may happen that two different truth values are contained in the proof and the weaker one is used. We then modify the proof to ensure updating. As a result, the truth value $s$ associated to $\gamma$ as well as the truth value associated to the rule's conclusion, are enlarged. The claim follows by induction.

Theorem 4.9. Let $\mathcal{T}$ be an assumption-preserving consistent theory of $\mathrm{CadL}$ and $\varphi$ be atomic. Assume that there is a sharp-first, regular proof of $(\varphi, t)$ from $\mathcal{T}$. Then there is a $t^{\prime} \succcurlyeq t$ and a sharp-first, regular proof of $\left(\varphi, t^{\prime}\right)$ from $\mathcal{T}$ which is also updating.

Proof. If $t$ is sharp, by Lemma 4.4 there is a proof containing only sharp propositions. This proof is sharp-first, updating, and also regular.

Let $t$ be fuzzy. A sharp-first proof fulfils (Up1'); so the assertion follows from Lemma 4.8 .

Let us summarise what we have established. By the results of the last section, we know that a run of CADIAG-2 can be emulated by a proof in CadL and an 
updating proof in CadL can be translated to a run of CADIAG-2. Theorem 4.9 permits to drop the "updating" condition under certain assumptions: Sharp-first regular proofs from assumption-preserving, consistent theories are updating and thus can be translated to a run of CADIAG-2. Recall that a proof to be sharp-first means that there is a first part whose argumentation is like in classical logic and a second part where comes everything properly fuzzy. In a regular proof, moreover, the rule introducing negation may be used only if the truth value of the assumption is known exactly.

\section{A Gentzen-style proof system for CadL}

In this section we formulate an alternative calculus reflecting the reasoning of CADIAG-2. In contrast to CadL it allows a semantical foundation: all rules are sound with respect to the semantics mentioned in the preceding chapter. Furthermore, the new calculus is formulated as a Gentzen-style system, cf. [Gen].

The introduced calculus, which we call SeqCadL, does not coincide with CadL; however, when restricting to regular proofs in CadL the two calculi do prove the same.

As we will see, SeqCadL allows a clear comparison with fuzzy logics in the sense of [Haj].

Definition 5.1. The atomic propositions of SeqCadL are countably many symbols $\varphi_{1}, \varphi_{2}, \ldots$ (to which we will sometimes refer to as variables) as well as constants $\bar{t}$ for each rational $t \in[0,1]$. The lattice propositions of SeqCadL consist of the expressions built up from the atomic propositions by means of the binary connectives $\wedge$ and $\vee$ and the unary connectives $\sim$ and $\Delta$ (see [Baa]).

Moreover, a $c$-sequent is of one of the following forms:

$$
\bar{t} \Rightarrow \alpha, \quad \alpha \Rightarrow \bar{t}, \quad \bar{t} \wedge \alpha \Rightarrow \beta, \quad \bar{t} \Rightarrow \Delta \alpha
$$

where $\bar{t}$ is a rational truth constant and $\alpha, \beta$ are lattice propositions.

An evaluation of SeqCadL maps the lattice propositions to $[0,1]$ such that (i) $v(\bar{t})=t$ for each rational $t \in[0,1]$, (ii) $v(\alpha \wedge \beta)=v(\alpha) \bar{\wedge} v(\beta)$, (iii) $v(\alpha \vee \beta)=$ $v(\alpha) \bar{v} v(\beta)$, (iv) $v(\sim \alpha)=\bar{\sim} v(\alpha)$, (v) $v(\Delta \alpha)=\bar{\Delta} v(\alpha)$, where

$$
\bar{\Delta} t= \begin{cases}1 & \text { if } t=1, \\ 0 & \text { else }\end{cases}
$$

for $t \in[0,1]$. 
An evaluation $v$ satisfies the c-sequent $\alpha \Rightarrow \beta$ if $v(\alpha) \leq v(\beta)$.

A theory $\mathcal{T}$ of SeqCadL is a finite set of c-sequents. We say that a theory $\mathcal{T}$ semantically implies a c-sequent $\alpha \Rightarrow \beta$ if any evaluation satisfying every element of $\mathcal{T}$ satisfies $\alpha \Rightarrow \beta$ as well.

We next define a proof system for SeqCadL. A SeqCadL rule is a pair consisting of a finite set of assumptions, i.e. a finite (possibly empty) set of c-sequents, and a conclusion. Rules with no assumptions are called axioms.

Definition 5.2. The rules of SeqCadL are, for any $\alpha, \beta \in \mathcal{F}_{L}$ and $s, t, d \in[0,1]$, the following:

$$
\begin{aligned}
& \overline{\overline{0} \Rightarrow \alpha}(0 \leq) \quad \overline{\alpha \Rightarrow \overline{1}}(\leq 1) \\
& \frac{\bar{s} \Rightarrow \alpha \quad \bar{t} \Rightarrow \beta}{\overline{s \bar{\lambda} t} \Rightarrow \alpha \wedge \beta}(\Rightarrow \wedge) \frac{\alpha \Rightarrow \bar{s} \quad \beta \Rightarrow \bar{t}}{\alpha \wedge \beta \Rightarrow \overline{s \bar{\wedge} t}}(\wedge \Rightarrow) \\
& \frac{\alpha \Rightarrow \overline{0}}{\alpha \wedge \beta \Rightarrow \overline{0}}\left(\wedge_{1} \Rightarrow 0\right) \frac{\beta \Rightarrow \overline{0}}{\alpha \wedge \beta \Rightarrow \overline{0}}\left(\wedge_{2} \Rightarrow 0\right) \\
& \frac{\alpha \Rightarrow \bar{s} \quad \beta \Rightarrow \bar{t}}{\alpha \vee \beta \Rightarrow \overline{s \overline{ } t}}(\vee \Rightarrow) \quad \frac{\bar{t} \Rightarrow \alpha}{\bar{t} \Rightarrow \alpha \vee \beta}\left(\Rightarrow \vee_{1}\right) \quad \frac{\bar{t} \Rightarrow \beta}{\bar{t} \Rightarrow \alpha \vee \beta}\left(\Rightarrow \vee_{2}\right) \\
& \frac{\bar{t} \Rightarrow \alpha}{\sim \alpha \Rightarrow \overline{\sim t}}\left(\sim_{1}\right) \quad \frac{\alpha \Rightarrow \bar{t}}{\overline{\bar{\tau} t} \Rightarrow \sim \alpha}\left(\sim_{2}\right) \quad \frac{\overline{1} \Rightarrow \sim \alpha}{\alpha \Rightarrow \overline{0}}\left(\sim_{3}\right) \quad \frac{\alpha \Rightarrow \overline{0}}{\overline{\overline{1}} \Rightarrow \sim \alpha}\left(\sim_{4}\right) \\
& \frac{\overline{1} \Rightarrow \alpha}{\overline{1} \Rightarrow \Delta \alpha}(\Delta) \quad \frac{\bar{t} \Rightarrow \alpha \quad \bar{d} \wedge \alpha \Rightarrow \beta}{\overline{d \bar{\wedge} t} \Rightarrow \beta}(\text { Cut }), \text { where } t, d>0
\end{aligned}
$$

A proof of a c-sequent $\alpha \Rightarrow \beta$ from a theory $\mathcal{T}$ is a labelled tree whose root is labelled by $\alpha \Rightarrow \beta$, the leaves are labelled by $(0 \leq),(\leq 1)$ or by a c-sequent in $\mathcal{T}$ and the inner nodes are labelled in accordance with instances of the rules.

We immediately see that SeqCadL is sound, that is, if the assumption of a rule (if present) is satisfied by some evaluation, then so is the conclusion:

Proposition 5.3. Let $\mathcal{T}$ be a theory of SeqCadL and let $\alpha \Rightarrow \beta$ be a c-sequent provable from $\mathcal{T}$. Then $\mathcal{T}$ semantically implies $\alpha \Rightarrow \beta$. 
Let $\mathcal{T}_{v}$ be the theory of CadL associated to the initial evaluation $v$ of CADIAG-2 (cf. Section 3). We denote by $\mathcal{T}_{v}^{s}$ the theory of SeqCadL obtained by translating each element of $\mathcal{T}_{v}$ as follows:

(i) To each $(\varphi, t)$, we associate $\bar{t} \Rightarrow \varphi$ and $\varphi \Rightarrow \bar{t}$.

(ii) To each implication $(\alpha \rightarrow \beta, d)$, where $\beta$ is atomic, we associate $\bar{d} \wedge \alpha \Rightarrow \beta$.

(iii) To each implication $(\alpha \rightarrow \sim \beta, 1)$, where $\beta$ is atomic, we associate $\overline{1} \wedge \Delta \alpha \Rightarrow$ $\sim \beta$.

Note that in case (ii), the implication necessarily originates from a rule of type (c) in the knowledge base of CADIAG-2, whereas in case (iii), the implication either originates from a rule of type (ao) or (me).

Theorem 5.4 (CadL vs. SeqCadL). Let $\mathcal{T}$ be the theory of CadL associated to some initial evaluation of CADIAG-2. If there is a regular proof of $(\alpha, t)$ from $\mathcal{T}$ in CadL, then SeqCadL proves $\bar{t} \Rightarrow \alpha$ ift $>0$ and $\alpha \Rightarrow \overline{0}$ if $t=0$, from $\mathcal{T}^{s}$.

Proof. Let $P$ be a regular proof in CadL from $\mathcal{T}_{v}$. Translate each entry in $P$ of the form $(\beta, s)$, where $s>0$, to $\bar{s} \Rightarrow \beta$; each entry in $P$ of the form $(\beta, 0)$ to $\beta \Rightarrow \overline{0}$; and each implication as shown above.

The result is not necessarily a proof in SeqCadL; modifications might be necessary. For $i \geq 1$, assume that the first $i-1$ proof entries do form a proof in SeqCadL. If the $i$-th proof entry is, in the original CadL proof, derived by an evaluation rule different from $(\sim)$, there is a corresponding rule in SeqCadL. If $(\sim)$ is used with sharp truth values, there are the corresponding rules $\left(\sim_{1}\right)$ and $\left(\sim_{2}\right)$.

If $(\sim)$ is used with non-sharp truth values, then, by regularity, for every atom $\varphi$ in the assumption, $(\varphi, s)$ is contained in $\mathcal{T}_{v}$ if $s$ is non-sharp, else in the preceding part of the proof; hence both $\bar{s} \Rightarrow \varphi$ and $\varphi \Rightarrow \bar{s}$ are available. We conclude that we may extend the proof to prove the assumption of $(\sim)$ with antecedent and succedent interchanged.

Finally, let one of the manipulation rules be used to derive the $i$-th proof entry. (c) corresponds to (Cut) in SeqCadL. The rule (ao) can be simulated in SeqCadL as 
follows; note that $\overline{1 \bar{\wedge} 1}$ is $\overline{1}$ :

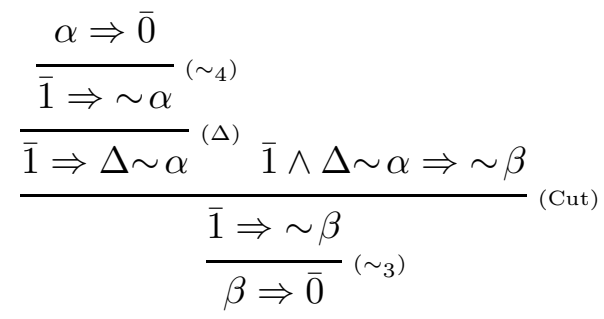

The case of the rule (me) is similar.

Theorem 5.5 (SeqCadLvs. CadL). Let $\mathcal{T}$ be the theory of CadL associated to some initial evaluation of CADIAG-2. If there is a proof in SeqCadL of $\bar{u} \Rightarrow \delta$ where $u>0$, or $\delta \Rightarrow \overline{0}$, from $\mathcal{T}^{s}$, then $\mathrm{CadL}_{\text {proves }}(\delta, u)$ or $(\delta, 0)$, respectively, from $\mathcal{T}$.

Proof. Let a proof of SeqCadL be given. We first translate the proof steps as follows: $\mathrm{c}$-sequents of the form $\overline{1} \Rightarrow \sim \alpha$ to $(\alpha, 0)$ and $\bar{t} \Rightarrow \alpha$ or $\alpha \Rightarrow \bar{t}$ to $(\alpha, t)$; c-sequents of the form $\bar{d} \wedge \alpha \Rightarrow \beta$ are translated to their original versions in accordance to (ii)-(iii) above.

We consider successively each proof step and make modifications where necessary. The rules $(0 \leq),(\leq 1),\left(\sim_{3}\right),\left(\sim_{4}\right)$, and $(\Delta)$ are ignored. If a proof step is based on a rule for $\wedge$ or $\vee$ or on the rule $\left(\sim_{1}\right)$ or $\left(\sim_{2}\right)$, we use the corresponding rules of CadL.

Consider now an application of (Cut). Note that the right premise of (Cut) is necessarily contained in $\mathcal{T}^{s}$ because there is no way to derive a c-sequent of this form from a rule; therefore the right premise is the translation of an implication from $\mathcal{T}$. Two cases can arise. The first possibility, corresponding to case (ii) above,

$$
\frac{\bar{t} \Rightarrow \alpha \quad \bar{d} \wedge \alpha \Rightarrow \beta}{\overline{d \overline{ } t} \Rightarrow \beta}
$$

translates to a rule (c). For the second possibility, corresponding to case (iii) above, note that a c-sequent of the form $\bar{t} \Rightarrow \Delta \alpha$, is always derived by means of $(\Delta)$, and in particular we always have $t=1$. Thus

$$
\frac{\overline{1} \Rightarrow \Delta \alpha \quad \overline{1} \wedge \Delta \alpha \Rightarrow \beta}{\overline{1} \Rightarrow \sim \beta}
$$

translates to the rule (me) or (ao). 


\section{Fuzzy Logics and SeqCadL}

In this section we compare SeqCadL with the t-norm based logic closest to the natural semantical framework for CadL. Not surprisingly, the logic which we have in mind is an extension of Gödel logic. More precisely we consider a variant of the logic $\mathrm{RGL}_{\sim}$, which was introduced in [EGHN] as Gödel logic enriched by the standard negation as well as rational truth constants.

First note that the objects of SeqCadL, i.e. the c-sequents, have the form $\alpha \Rightarrow$ $\beta$, where $\alpha$ and $\beta$ do not contain the implication symbol. Accordingly, we are going to consider (a variant of) the fragment of $R G L_{\sim}$ with no nested implications. For the similarity to the proposal of Zadeh in [Zad], we call the resulting logic Gödel-Zadeh logic, GZL for short. Note however that we include Baaz's $\Delta$ in the language.

Definition 6.1. The atomic and lattice propositions of GZL are defined like for SeqCadL (see Definition 5.1). A comparing proposition of GZL is a pair of two lattice propositions $\alpha$ and $\beta$, notated by $\alpha \rightarrow \beta$.

An evaluation of GZL is defined like for SeqCadL. We say that an evaluation $v$ satisfies a comparing proposition $\alpha \rightarrow \beta$ if $v(\alpha) \leq v(\beta)$.

A theory of GZL is a set of comparing propositions. We say that a theory $\mathcal{T}$ semantically implies a proposition $\alpha \rightarrow \beta$ if any evaluation satisfying every element of $\mathcal{T}$ satisfies $\alpha \rightarrow \beta$ as well.

A proof system for GZL is formulated below using sequents-of-relations, a generalisation of Gentzen sequents introduced in $[\mathrm{BaFe}]$ in which the sequent arrow " $\Rightarrow$ " is splitted into the inequality symbols " $\leq$ " and " $<$ ". Our calculus extends that for Gödel logic with $\Delta$ in $[\mathrm{BCF}]$ with suitable rules for negation and truth constants.

In our context, a sequent-of-relations $\mathcal{G}$ is a multiset of ordered triples

$$
\alpha_{1} \triangleleft_{1} \beta_{1}|\ldots| \alpha_{n} \triangleleft_{n} \beta_{n},
$$

where $\alpha_{i}$ and $\beta_{i}$ are formulas of GZL and $\triangleleft_{i} \in\{<, \leq\}$ for $i=1, \ldots, n$. Note that the use of inequality symbols " $\leq$ " and " $<$ " in the definition is purely syntactic (although of course also suggestive of the intended meaning).

$\mathcal{G}$ is satisfied by some evaluation of GZL $v$ if $v\left(\alpha_{i}\right) \triangleleft_{i} v\left(\beta_{i}\right)$ for some $i$; $\mathcal{G}$ is valid in GZL if satisfied by all evaluation of GZL.

A literal is a lattice proposition of the form $\varphi$ or $\sim \varphi$ for some atom $\varphi$. We call a sequent-of-relations basic if it contains only literals. 
Definition 6.2. Axioms and rules of SeqGZL are the following, where $\mathcal{G}$ is an arbitrary side sequent-of-relations and $\triangleleft, \triangleleft_{1}, \triangleleft_{2} \in\{<, \leq\}$ :

Axioms

$$
\overline{\alpha \leq \alpha}(A 1) \quad \overline{\bar{s} \triangleleft \bar{t}}(A 2) \text {, where } s \triangleleft t
$$

\section{Logical Rules}

$$
\begin{gathered}
\frac{\mathcal{G}|\alpha \triangleleft \gamma| \beta \triangleleft \gamma}{\mathcal{G} \mid \alpha \wedge \beta \triangleleft \gamma}(\wedge \triangleleft) \quad \frac{\mathcal{G}|\gamma \triangleleft \alpha \quad \mathcal{G}| \gamma \triangleleft \beta}{\mathcal{G} \mid \gamma \triangleleft \alpha \wedge \beta}(\triangleleft \wedge) \\
\frac{\mathcal{G}|\alpha \triangleleft \gamma \quad \mathcal{G}| \beta \triangleleft \gamma}{\mathcal{G} \mid \alpha \vee \beta \triangleleft \gamma}(\vee \triangleleft) \quad \frac{\mathcal{G}|\gamma \triangleleft \alpha| \gamma \triangleleft \beta}{\mathcal{G} \mid \gamma \triangleleft \alpha \vee \beta}(\triangleleft \vee) \\
\frac{\mathcal{G} \mid \sim \beta \triangleleft \alpha}{\mathcal{G} \mid \sim \alpha \triangleleft \beta}(\sim \triangleleft) \quad \frac{\mathcal{G} \mid \beta \triangleleft \sim \alpha}{\mathcal{G} \mid \alpha \triangleleft \sim \beta}(\triangleleft \sim) \quad \frac{\mathcal{G} \mid \beta \triangleleft \alpha}{\mathcal{G} \mid \sim \alpha \triangleleft \sim \beta}(\sim \triangleleft \sim) \\
\frac{\mathcal{G}|\alpha<1| 1 \leq \beta}{\mathcal{G} \mid \Delta \alpha \leq \beta}(\Delta \leq) \quad \frac{\mathcal{G}|\alpha \leq 0| 1 \leq \beta}{\mathcal{G} \mid \alpha \leq \Delta \beta}(\leq \Delta) \\
\frac{\mathcal{G} \mid \alpha<1}{\mathcal{G} \mid \Delta \alpha<\beta}(\Delta<) \quad \frac{\mathcal{G} \mid \alpha<1}{\mathcal{G} \mid \alpha<\Delta \beta}(<\Delta)
\end{gathered}
$$

Rules for Constants

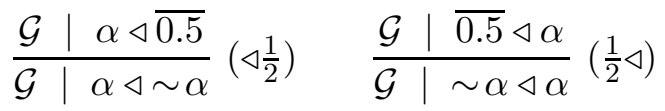

$$
\begin{aligned}
& \frac{\mathcal{G} \mid \alpha \triangleleft \overline{0}}{\mathcal{\mathcal { G }} \mid \alpha \triangleleft \beta}(\triangleleft 0) \quad \frac{\mathcal{G} \mid \overline{1} \triangleleft \beta}{\mathcal{G} \mid \alpha \triangleleft \beta}(1 \triangleleft)
\end{aligned}
$$

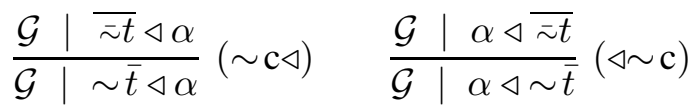


Structural Rules

$$
\begin{gathered}
\frac{\mathcal{G}|\alpha \triangleleft \beta| \alpha \triangleleft \beta}{\mathcal{G} \mid \alpha \triangleleft \beta}(\mathrm{EC}) \quad \frac{\mathcal{G}|\alpha \leq \beta \quad \mathcal{G}| \gamma \triangleleft_{1} \delta}{\mathcal{G}\left|\gamma \triangleleft_{1} \beta\right| \alpha \triangleleft_{2} \delta}(\mathrm{com}) \\
\frac{\mathcal{G}}{\mathcal{G} \mid \alpha \triangleleft \beta}(\mathrm{EW}) \quad \frac{\mathcal{G}|\alpha<\beta \quad \mathcal{G}| \beta \leq \alpha}{\mathcal{G}}(\mathrm{cut})
\end{gathered}
$$

The definition of proof in SeqGZL is as usual.

Lemma 6.3. In SeqGZL, the rule

$$
\frac{\mathcal{G}|\alpha \leq \beta \quad \mathcal{G}| \beta \leq \gamma}{\mathcal{G} \mid \alpha \leq \gamma} \text { (trans) }
$$

is derivable.

Proof.

$$
\frac{\mathcal{G}|\beta \leq \gamma \quad \mathcal{G}| \alpha \leq \beta}{{ }_{(\mathrm{com})} \mathcal{G} \mid \beta \leq \beta}{ }_{(\mathrm{cut})}
$$

Remark 6.4. Note that although we deal with comparing propositions, rules for implication are not needed. Corollary 6.7 below shows how the two arrows can replace these rules.

Remark 6.5. Note that the methodology introduced in [BaFe] to define a sequentof-relations calculus out of the semantic specification of a logic does not work for SeqGZL whose semantics, due to the presence of a classical (involutive) negation, is not projective.

As usual we will say that a rule is sound in GZL if whenever all assumptions are satisfied by some evaluation $v$ of GZL, the conclusion is satisfied by $v$ as well. A rule is invertible if whenever the conclusion is valid, then so are all assumptions.

Theorem 6.6. A sequent-of-relations $\mathcal{G}$ is valid in $\mathrm{GZL}$ if and only if $\mathcal{G}$ is provable in SeqGZL without use of the rule (Cut). 
Proof. It is easy to see that the rules of SeqGZL are sound.

For completeness, observe that the axioms are valid and logical rules are invertible. Moreover when not allowing the subsequent use of the negation rules $(\sim \triangleleft)$ and $(\triangleleft \sim)$, inverse (i.e. bottom up) applications of the logical rules lead to basic sequents-of-relations in a finite number of steps.

It remains to show that SeqGZL derives all basic sequents-of-relations which are valid. Let $\mathcal{G}$ be a valid basic sequent-of-relations. We proceed by induction on the number of variables in $\mathcal{G}$. If there is no variable, we remove any appearance of the $\sim$-connective by inverse application of $(\sim \mathrm{c} \triangleleft)$ or $(\triangleleft \sim \mathrm{c})$. The result must contain a sequent of the form $\bar{s} \triangleleft \bar{t}$, where $s \triangleleft t$, and it is derivable by (A2) and (EW).

Let $\mathcal{G}$ contain at least one variable, say $\alpha$. We show that $\mathcal{G}$ can be derived from a valid sequent-of-relations $\mathcal{G}^{\prime}$ whose variables are those which already occur in $\mathcal{G}$, but not $\alpha$.

By backwards application of the rules for $\sim$ if necessary, in a basic sequents-ofrelations the possible relations involving $\alpha$ are of one of the following forms: (i) $\alpha \triangleleft \beta$ for some literal $\beta$ not containing $\alpha$, or (ii) of the form $\gamma \triangleleft \alpha$ for some literal $\gamma$ not containing $\alpha$, or (iii) $\alpha$ occurs on both sides of $\triangleleft$.

Case (iii) can be discarded. Indeed, if $\alpha \leq \alpha$ appears, then $\mathcal{G}$ is derivable by (A1) and (EW). If $\mathcal{G}=\mathcal{G}^{\prime} \mid \alpha<\alpha$, then $\mathcal{G}$ is valid if and only if so is $\mathcal{G}^{\prime}$, and $\mathcal{G}$ is derivable from $\mathcal{G}^{\prime}$ by (EW). Furthermore $\sim \alpha \triangleleft \alpha$ and $\alpha \triangleleft \sim \alpha$ can be replaced by $\overline{0.5} \triangleleft \alpha$ or $\alpha \triangleleft \overline{0.5}$, respectively, by means of the $\frac{1}{2}$-rules.

Assume next that case (i) occurs in $\mathcal{G}$, but not (ii). Then we replace all occurrences of $\alpha$ by $\overline{1}$; from the result, which is evidently still valid, we derive $\mathcal{G}$ by $(1 \triangleleft)$. We proceed similarly if only case (ii) occurs.

Assume finally that cases (i) and (ii) appear both at least once. We assume that they appear exactly twice; otherwise the argumentation is similar. Let $\beta_{1} \triangleleft \alpha, \beta_{2} \triangleleft \alpha$, $\alpha \triangleleft \gamma_{1}, \alpha \triangleleft \gamma_{2}$ occur in $\mathcal{G}$, where $\triangleleft$ is chosen as $\leq$ or $<$ independently in the four cases. Then we build $\mathcal{G}^{\prime}$ by replacing these four relations by $\beta_{1} \triangleleft \gamma_{1}, \beta_{1} \triangleleft \gamma_{2}, \beta_{2} \triangleleft \gamma_{1}$, $\beta_{2} \triangleleft \gamma_{2}$, where $\triangleleft$ is $<$ only if the involved atoms both have a $<$-relation with $\alpha$. Then $\mathcal{G}$ is derivable from $\mathcal{G}^{\prime}$ by (com) and (EC). Furthermore, $\mathcal{G}^{\prime}$ is valid; indeed, if there is an evaluation making every relation in $\mathcal{G}^{\prime}$ fail, we can extend it to $\alpha$ to make every relation in $\mathcal{G}$ fail.

Corollary 6.7. Let $\mathcal{T}=\left\{\alpha_{1} \rightarrow \beta_{1}, \ldots, \alpha_{n} \rightarrow \beta_{n}\right\}$ be a finite theory of GZL and let $\alpha \rightarrow \beta$ be a comparing proposition of GZL. $\mathcal{T}$ semantically implies $\alpha \rightarrow \beta$ if and only if there is a proof in SeqGZL of $\alpha \leq \beta$ from $\left\{\alpha_{1} \leq \beta_{1}, \ldots, \alpha_{n} \leq \beta_{n}\right\}$

Proof. By Theorem 6.6 there is a proof in SeqGZL of the sequent-of-relations $\beta_{1}<$ 
$\alpha_{1}|\ldots| \beta_{n}<\alpha_{n} \mid \alpha \leq \beta$. The claim follows by $n$ applications of (cut) with premises $\alpha_{i} \leq \beta_{i}$, with $i=1, \ldots, n$.

We next show that GZL is stronger than SeqCadL and hence of CadL.

Lemma 6.8. Let $\mathcal{T}$ be a theory of SeqCadL, $\mathcal{T}^{\prime}$ be the corresponding theory of SeqGZL and $\alpha \Rightarrow \beta$ be a c-sequent of SeqCadL. If $\alpha \Rightarrow \beta$ is provable in SeqCadL from $\mathcal{T}$ then $\alpha \leq \beta$ is provable in SeqGZL from $\mathcal{T}^{\prime}$.

Proof. We have to show that every rule of SeqCadL in which the symbol $\Rightarrow$ is replaced by $\leq$ is derivable in SeqGZL. This is easy in case of all rules but (Cut). To derive (Cut), we consider the two cases $t \leq d$ and $d \leq t$. If $t \leq d$, we may derive in SeqGZL (we omit side sequents-of-relations):

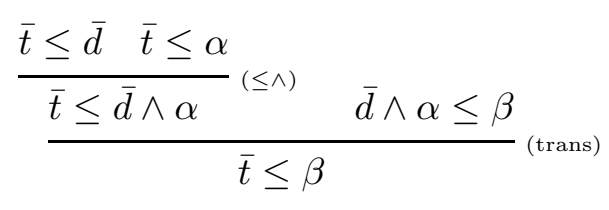

If $d \leq t$, we have

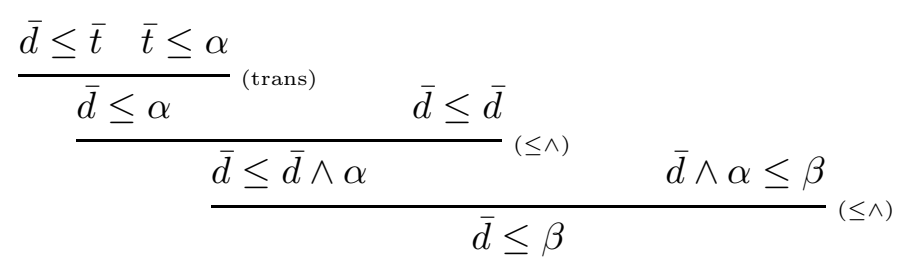

In view of Theorem 5.4, we get as a corollary that SeqGZL is able to reproduce all inferences of CADIAG-2 modelled by regular proofs in CadL.

Note that the converse of Lemma 6.8 does not hold. Indeed, we have:

Lemma 6.9. Let $\mathcal{T}=\{\overline{1} \wedge \alpha \Rightarrow \beta, \overline{1} \wedge \sim \alpha \Rightarrow \beta\}$, where $\alpha$ and $\beta$ are atoms, be a theory of SeqCadL. Then SeqCadL does not prove $\overline{0.5} \Rightarrow \beta$ from $\mathcal{T}$.

Proof. If a proof of $\overline{0.5} \Rightarrow \beta$ from $\mathcal{T}$ exists, then by the soundness of SeqCadL it must necessarily contain an element of $\mathcal{T}$, say $\overline{1} \wedge \alpha \Rightarrow \beta$. A sequent of this form appears only as the premise of the rule (Cut); the first one is $\bar{t} \Rightarrow \alpha$ for some $t \in[0,1]$. Again by the soundness of SeqCadL, $\bar{t} \Rightarrow \alpha$ is derivable from $\mathcal{T}$ in SeqCadL only if $t=0$. But then the conclusion of (Cut) is $\overline{0} \Rightarrow \beta$, which is derivable from the empty theory already. The assertion follows. 
Corollary 6.10. SeqGZL is strictly stronger than SeqCadL.

Proof. The assertion follows from the previous lemma and the fact that from $\alpha \leq \beta$ and $\sim \alpha \leq \beta$ we can derive $\overline{0.5} \leq \beta$ in SeqGZL. Indeed, we have

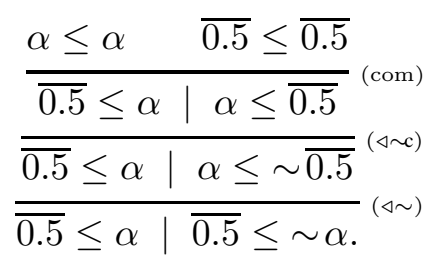

Applying twice (com) and then (EC), we furthermore derive $\overline{0.5} \leq \beta|\beta<\alpha|$ $\beta<\sim \alpha$. We finally apply twice (cut), and the assertion follows.

Therefore SeqGZL, and hence by Theorem 6.6 GZL, is not adequate to formalise CADIAG-2. An informal question is what GZL can do more. The crucial fact responsible for the additional strength of SeqGZL is the total order of the set of truth constants. As a consequence, it is necessary to base a Gentzen-style calculus for GZL on sequents-of-relations rather than on single relations. In the above example, it is necessary to express in some way the alternative " $\alpha \leq \beta$ or $\beta \leq \alpha$ ", done in SeqGZL by the provable sequent-of-relations

$$
\alpha \leq \beta \mid \beta \leq \alpha,
$$

which has no counterpart in SeqCadL.

So comparing the two calculi SeqGZL and SeqCadL, we may certainly observe a number of technical differences between the two systems on the lowest level. But when abstracting from details and considering just the crucial point of difference, we have that SeqGZL is based on sequents-of-relations and SeqCadL just on single relations. We may state: SeqGZL and SeqCadL are based on the same semantics; however, only SeqGZL allows to consider alternatives concerning the relationship between pairs of propositions. Referring to the example given above, SeqCadL does not allow to argue: "Either property 1 holds stronger than property 2, or property 2 holds stronger than property 1"; SeqCadL does not support this kind of case-based reasoning.

An explanation of this phenomenon can be rather easily found. SeqCadL deals with statements which either specify the strength of some property explicitly "The property $\varphi$ holds at least to the degree 0.9 ", or it uses statements which express causal or logical relationships: "Property 1 implies property 2." To speak about a causal implication between two facts, however, is different from speaking about the strength of some property compared to another one. 
We conclude that the medical expert system CADIAG-2 is based on principles very close to the basic principles known from t-norm based logics; as a consequence, we may emulate the inference mechanism of the system by means of a familiar fuzzy-logical calculus. But there remain differences on a basic level that cannot be easily overcome.

\section{Acknowledgements}

This research was partially supported by the Vienna Science and Technology Fund (WWTF) Grant MA07-016.

We are indebted to K.-P. Adlassnig for intensive discussions about CADIAG-2. We also thank P. Rusnok for helping with the examples taken from the CADIAG-2 knowledge base, and G. Metcalfe for providing us the key idea for a short proof of Theorem 6.6. We would furthermore like to thank the anonymous referees whose comments helped to improve the paper.

\section{References}

[AdKo] K.-P. Adlassnig, G. Kolarz, CADIAG-2: Computer-Assisted Medical Diagnosis Using Fuzzy Subsets, in: M. M. Gupta, E. Sanchez (eds.), "Approximate Reasoning in Decision Analysis", North-Holland Publ. Comp., Amsterdam 1982; 219 - 247.

[AKSEG] K.-P. Adlassnig, G. Kolarz, W. Scheithauer, H. Effenberger, G. Grabner, CADIAG: Approaches to computer-assisted medical diagnosis, Comput. Biol. Med. 15 (1985), 315 - 335.

[AKSG] K.-P. Adlassnig, G. Kolarz, W. Scheithauer, G. Grabner, Approach to a hospital-based application of a medical expert system, Med. Inform. 11 (1986), $205-223$.

[Baa] M. Baaz, Infinite-valued Gödel logics with 0-1-projections and relativizations, in: P. Hájek (ed.), "Gödel '96. Logical foundations of mathematics, computer science and physics - Kurt Gödel's legacy”, SpringerVerlag, Berlin 1996; 23 - 33.

[BaFe] M. Baaz, C. G. Fermüller, Analytic Calculi for Projective Logics, in: N. V. Murray (ed.), "Automated reasoning with analytic tableaux and related methods", Proceedings of TABLEAUX '99, Springer-Verlag, Berlin 1999; 36 - 50. 
[BCF] M. Baaz, A. Ciabattoni, C. G. Fermüller, Sequent of Relations Calculi: a Framework for Analytic Deduction in Many-Valued Logics, in: M. Fitting, E. Orlowska (eds.), "Beyond Two: Theory and applications of Multiple-Valued Logics”, Physica-Verlag, Heidelberg 2003; 152 - 175.

[BAHRL] K. Boegl, K.-P. Adlassnig, Y. Hayashi, T. E. Rothenfluh, H. Leitich, Knowledge Acquisition in the Fuzzy Knowledge Representation Framework of a Medical Consultation System, Artificial Intelligence in Medicine 30 (2004), 1 - 26.

[EGHN] F. Esteva, Ll. Godo, P. Hájek, M. Navara, Residuated fuzzy logics with an involutive negation, Arch. Math. Logic 39 (2000), 103 - 124.

[DHN] M. Daniel, P. Hájek, P. H. Nguyen, CADIAG-2 and MYCIN-like systems. Artificial Intelligence in Medicine 9 (1997), 241-259.

[DEGP] D. Dubois, F. Esteva, L. Godo, H. Prade. Fuzzy-set based logics - an history-oriented presentation of their main developments, in: D. M. Gabbay J. Woods (eds.), "Handbook of The History of Logic", vol. 8, Elsevier, Amsterdam 2007; 325 - 449.

[Fis] F. Fischler, "Die Wissensbasis und der Inferenzprozeß des medizinischen Expeteretnsystems CADIAG-II/E", Diploma thesis, Medical University of Vienna, Vienna 1994.

[GJEHH] J. S. Garner, W. R. Jarvis, T. G. Emori, T. C. Horan, J. M. Hughes, CDC definitions for nosocomial infections. In: R. N. Olmsted (ed.), "APIC Infection Control and Applied Epidemiology: Principles and Practice", Mosby, St. Louis 1996; A-1-A-20.

[Gen] G. Gentzen, Untersuchungen über das logische Schließen I, II, Mathematische Zeitschrift 39 (1934), 176 - 210, 405 - 431.

[Ger] G. Gerla, "Fuzzy Logic", Kluwer, Dordrecht 2000.

[Haj] P. Hájek, "Metamathematics of Fuzzy Logic”, Kluwer, Dordrecht 1998.

[LAK] H. Leitich, K.-P. Adlassnig, G. Kolarz, Evaluation of Two Different Models of Semiautomatic Knowledge Acquisition for the Medical Consultant System CADIAG-II/RHEUMA, Artifical Intelligence in Medicine 25 (2002), 215 - 225.

[MoAd] W. Moser, K.-P. Adlassnig, Consistency checking of binary categorical relationships in a medical knowledge base, Artificial Inteligence in Medicine 7 (1992), 389 - 407. 
[NPM] V. Novák, I. Perfilieva, J. Močkoř, "Mathematical principles of fuzzy logic", Kluwer, Dordrecht 1999.

[Pav] J. Pavelka, On fuzzy logic I, II, III, Z. Math. Logik Grundl. Math. 25 (1979), 45 - 52, 119 - 134, 447 - 464.

[Zad] L. A. Zadeh, Fuzzy sets, Information and Control 8 (1965), 338353. 